OPEN ACCESS

Edited by:

Hari S. Misra,

Bhabha Atomic Research Centre

(BARC), India

Reviewed by:

Ravikumar Sambandam,

Aarupadai Veedu Medical College \&

Hospital, India

Harinder Singh,

SVKM's Narsee Monjee Institute

of Management Studies, India

*Correspondence:

Jiqian Wang

jqwang@upc.edu.cn

Hu Zhu

zhuhu@fjnu.edu.cn

Specialty section:

This article was submitted to

Microbial Physiology and Metabolism,

a section of the journa

Frontiers in Microbiology

Received: 10 October 2021

Accepted: 08 February 2022

Published: 01 March 2022

Citation:

Li H, Chen MQ, Zhang ZM, Li BC,

Liu JL, Xue H, Ji SX, Guo ZR,

Wang JQ and Zhu H (2022) Hybrid

Histidine Kinase WelA

of Sphingomonas sp. WG Contributes

to WL Gum Biosynthesis and Motility.

Front. Microbiol. 13:792315.

doi: 10.3389/fmicb.2022.792315

\section{Hybrid Histidine Kinase WelA of Sphingomonas sp. WG Contributes to WL Gum Biosynthesis and Motility}

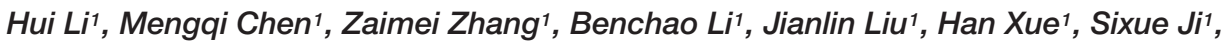 \\ Zhongrui Guo ${ }^{1}$, Jiqian Wang ${ }^{1 *}$ and Hu Zhu ${ }^{1,2 *}$ \\ ${ }^{1}$ State Key Laboratory of Heavy Oil Processing and Centre for Bioengineering and Biotechnology, China University \\ of Petroleum (East China), Qingdao, China, ${ }^{2}$ Engineering Research Center of Industrial Biocatalysis, Fujian Province \\ Universities, College of Chemistry and Materials Science, Fujian Normal University, Fuzhou, China
}

Sphingomonas sp. WG produced WL gum with commercial utility potential in many industries. A hybrid sensor histidine kinase/response regulator WelA was identified to regulate the WL gum biosynthesis, and its function was evaluated by gene deletion strategy. The WL gum production and broth viscosity of mutant $\triangle$ welA was only $44 \%$ and $0.6 \%$ of wild type strain at $72 \mathrm{~h}$. The transcriptomic analysis of differentially expressed genes showed that WelA was mapped to CckA; ChpT, and CtrA in the CckA-ChpT-CtrA pathway was up-regulated. One phosphodiesterase was up-regulated by CtrA, and the intracellular C-di-GMP was decreased. Most genes involved in WL gum biosynthesis pathway was not significantly changed in $\Delta$ welA except the upregulated atrB and atrD and the down-regulated $p m m$. Furthermore, the up-regulated regulators ctrA, flaEY, flbD, and flaF may participate in the regulation of flagellar biogenesis and influenced motility. These results suggested that CckA-ChpT-CtrA pathway and c-di-GMP were involved in WL gum biosynthesis regulation. This work provides useful information on the understanding of molecular mechanisms underlying WL gum biosynthesis regulation.

Keywords: Sphingomonas sp. WG, WelA, regulatory pathway, WL gum, c-di-GMP, flagellar assembly

\section{INTRODUCTION}

Exopolysaccharides (EPSs) secreted by many bacterial strains are ubiquitous and have a wide range of industrial applications, including emulsification, thickening, and pharmaceutical applications (Kumar et al., 2007; Freitas et al., 2011; Schmid et al., 2015). Several EPSs such as xanthan, gellan, curdlan, and succinoglucan have been commercially produced and applied in recent years (Lelchat et al., 2015). Sphingans such as gellan, S-88, welan, and diutan are EPSs produced by the genus Sphingomonas sp. They have a similar repeating tetrasaccharide backbone structure as glucoseglucuronic acid-glucose-rhamnose or mannose (Fialho et al., 2008). It has been proved that the known sphingans with special side chains have unique rheological characteristics and commercial applications in petroleum, food, concrete, and pharmaceutical industries as gelling, emulsifiers, stabilizers, and viscosifiers agents (Fialho et al., 2008). WL gum was one novel sphingan produced by the strain Sphingomonas sp. WG with high viscosity, pseudoplastic behavior, good resistance 
to high temperature and high concentration of salts, stability to a broad range of $\mathrm{pH}(1-14)$. As a result, it can be used in food, concrete, enhanced oil recovery, and many other industries (Li et al., 2016b).

Due to these valuable properties, studies were performed to improve the WL gum production. For example, the medium composition and fermentation parameters optimization strategy has been used, and the WL gum production reached $39.95 \mathrm{~g} / \mathrm{L}$, 2.37-fold of the initial production (16.82 g/L) (Li et al., 2018). However, the normal optimization of the fermentation process is restricted by the physiological limitation of strains. Therefore, metabolic engineering with increased sphingans production or volumetric productivity attracted more attention. A further understanding for the fundamental biosynthesis process and regulation mechanism is critical for genetic and metabolic engineering approaches. In our previous work, the genome of Sphingomonas sp. WG was sequenced and annotated (Li et al., 2016a), and the wel cluster for WL gum biosynthesis was predicted. Similar to the previously reported sphingans [gellan gum (Harding et al., 2004), S88 (Yamazaki et al., 1996), diutan gum (Coleman et al., 2008), and welan gum (Schmid et al., 2014)], the biosynthesis of WL gum might follow a Wzx/Wzy-dependent pathway and three sequential steps involved: (i) intracellular synthesis of nucleotide-sugar precursors catalyzed by PGM, UGP, UGD, etc.; (ii) assembly of the tetrasaccharide repeating units by different glycosyltransferases; (iii) polymerization of the assembled repeating units to form longer chains and export of the polysaccharide in the general Wzx/Wzy-dependent pathway. Our previous work has characterized the function of some enzymes, including PGM, UGP, and WelK (Li et al., 2019, 2021). The overexpression of a single gene or gene clusters for other sphingans was also employed to enhance the carbon flux toward the final polymer. However, it had a negative effect in many cases (Thorne et al., 2000; Sa-Correia et al., 2002). Therefore, the elucidation of the regulation mechanism of sphingan biosynthesis will allow the understanding of the bottlenecks of the sphingan biosynthesis pathway and provide the target for metabolic engineering.

The putative protein GelA, which is homologous to hybrid sensor kinase and response regulatory protein, was supposed to be involved in the gellan biosynthesis regulation because the mutants were non-mucoid on solid YM medium (Harding et al., 2004; Fialho et al., 2008). The over-expression of gelA in Sphingomonas elodea resulted in enhanced gellan biosynthesis, which might be related to the increased transportation of sugar units through comparative two-dimensional gel electrophoresis analysis (Lee et al., 2017). However, the signaling pathway of GelA in the regulation of gellan gum biosynthesis was still unclear. welA, a homologous version of gelA (78\% identity), is annotated in the draft genome of Sphingomonas sp. WG and proposed to encode the regulator for WL gum biosynthesis. Therefore, in this work, welA was knocked out from the genome of Sphingomonas sp. WG and its effects on WL gum production were investigated. Furthermore, the transcriptomic analysis was performed between the wild strain and the mutant strain with the aim of better understanding the metabolic pathways such as motility and WL gum biosynthesis regulated by WelA. The results obtained in this manuscript provided useful information on molecular mechanisms underlying the regulation of $\mathrm{WL}$ gum biosynthesis.

\section{MATERIALS AND METHODS}

\section{Strains, Plasmids, Culture Conditions, and Chemicals}

The strain Sphingomonas sp. WG (CCTCC No. M2013161) was maintained on Luria-Bertani (LB) agar slants at $4^{\circ} \mathrm{C}$ and was activated in seed medium $(10 \mathrm{~g} / \mathrm{L}$ glucose, $1 \mathrm{~g} / \mathrm{L}$ yeast extract (YE), $5 \mathrm{~g} / \mathrm{L}$ tryptone, $2 \mathrm{~g} / \mathrm{L} \mathrm{KH}_{2} \mathrm{PO}_{4}$, and $0.1 \mathrm{~g} / \mathrm{L} \mathrm{MgSO}_{4}$ ) at $28^{\circ} \mathrm{C}$. The activated strain was sequentially transferred to a $250 \mathrm{~mL}$ flask containing $50 \mathrm{~mL}$ fermentation medium $(67 \mathrm{~g} / \mathrm{L}$ glucose, $3.4 \mathrm{~g} / \mathrm{L}$ YE, 3 g/L K $\mathrm{HPO}_{4}, 0.1$ g/L MgSO 4 , pH 7.0; Zhou et al., 2017), and incubated at $32^{\circ} \mathrm{C}, 150 \mathrm{rpm}$ for $72 \mathrm{~h}$ to produce WL gum. To amplify the recombinant plasmid DNA, Escherichia coli $\mathrm{DH} 5 \alpha$ was chosen as the host strain, and related recombinant E. coli $\mathrm{DH} 5 \alpha$ strains were the donor strains in bacterial conjugation. $E$. coli $\mathrm{DH} 5 \alpha$ was cultivated at $37^{\circ} \mathrm{C}$ in $\mathrm{LB}$ medium supplemented with tetracycline or gentamicin when necessary. The plasmid pJQ200SK containing the $s a c B$ gene as the counter-selectable marker (Pelicic et al., 1996) was applied to construct the welA deletion strain $\triangle$ welA. The plasmid pBBR1MCS-3 (Kovach et al., 1995) was used to introduce welA gene into the $\triangle$ welA to construct the complemented strain $\triangle$ welA/pBBR1MCS-3-welA. The plasmid pET32a ${ }^{(+)}$and E. coli BL21(DE3) were used to express the possible diguanylate phosphodiesterase encoded by Gene 1094. Both plasmids pJQ200SK and pBBR1MCS-3 were obtained from Wuhan Miaoling Bioscience and Technology Co., Ltd. Enzymes used in molecular cloning, including restriction enzymes, LA Taq DNA polymerase, and the DNA Ligation Kit were obtained from TaKaRa Biotechnology (Dalian, China). KOD FX DNA Polymerase was purchased from TOYOBO (shanghai) Biotech Co., Ltd. Primers were synthesized by Shanghai Sangon Biotechnology (Shanghai, China). All antibiotics used were obtained from Sigma Chemical Co. (St. Louis, MO, United States). Other reagents were obtained from Sinopharm Chemical Reagent Co., Ltd. (Shanghai, China) and Shanghai Macklin Biochemical Co., Ltd.

\section{Bioinformatic Analysis of WelA}

Based on the amino acid similarity to GelA in gellan biosynthesis pathway (Harding et al., 2004), the gene welA (Gene 3707) was annotated on the whole genome of Sphingomonas sp. WG (GenBank no. LNOS00000000) (Li et al., 2016a) and its deduced protein WelA was obtained and analyzed by multiple bioinformatical tools. Firstly, the physical and chemical properties, hydrophilicity of WelA were predicted using ProtParam ${ }^{1}$ and ProtScale ${ }^{2}$ on the ExPASy Server (Wilkins et al., 1999). Furthermore, the related amino acid sequences were retrieved in the GenBank database using BlastP ${ }^{3}$ (Altschul et al., 1990) program, and the phylogenetic tree was constructed

\footnotetext{
${ }^{1}$ http://web.expasy.org/protparam/

${ }^{2}$ http://web.expasy.org/protscale/

${ }^{3}$ http://blast.ncbi.nlm.nih.gov/Blast.cgi
} 
using ClustalX version 2.0 (Larkin et al., 2007) and the MEGA7 program (Kumar et al., 2016). The secondary structure prediction and domain architecture analysis were performed by SOPMA ${ }^{4}$ (Geourjon and Deleage, 1995) and SMART ${ }^{5}$ (Letunic et al., 2015), respectively.

\section{Construction of the Mutants $\triangle w e l A$ and the Complemented Strain}

welA gene was knocked out from the chromosome of Sphingomonas sp. WG by double homologous recombination methods. Firstly, the upstream and downstream fragments (approximately 1,000 bp) of welA were amplified by PCR with primers welA5flFor/welA5flRev and welA3flFor/welA3flRev containing the tails with identity to the ends of each other (underlined) (Supplementary Table 1), respectively. Secondly, the obtained fragments were gel purified and fused into one DNA fragment with the help of their tails by fusing PCR reaction using "nested" primers welAdelFor/welAdelRev containing SacI and XbaI recognition sites (underlined). Thirdly, the resulting fusion product was inserted into the SacI$X b a$ I digested $\mathrm{pJQ} 200 \mathrm{SK}$ to obtain the recombinant plasmid pJQ200SK- $\triangle$ welA. Finally, the pJQ200SK- $\triangle$ welA was transferred into Sphingomonas sp. WG from E. coli DH5a by triparental conjugal mating with the help of E. coli pRK2013. The singlecrossover recombinants were screened by streptomycin and gentamicin. The deletion strains that undergo double-crossover recombination were screened on LB plates supplemented with $5 \%$ sucrose since single-crossover recombinants bearing the $s a c B$ gene are lethal in the presence of sucrose. The singlecrossover recombinants were identified by PCR using two pairs of primers welA5flFor/welAinRev and welAinFor/welA3flRev, and the deletion strain $\triangle w e l A$ was confirmed by PCR using welAinFor/welAinRev as primers.

A welA fragment was inserted into the plasmid pBBR1MCS3 to complement the welA gene. The ORF of welA was obtained by PCR using the Sphingomonas sp. WG genomic DNA as the template and welAexFor/welAexRev (The underlined parts were $X h o I$ and $X b a I$ recognition sties) as primers. Subsequentially, the gel-purified PCR product was digested by XhoI and XbaI and ligated into the linearized pBBR1MCS-3. The recombinant plasmid pBBR1MCS-3-welA was confirmed by colony PCR and DNA sequencing. Finally, the complemented strain of $\triangle$ welA with pBBR1MCS-3-welA was obtained by triparental conjugal mating and screened on LB supplemented with streptomycin and tetracycline. The complemented strain designated as $\triangle$ welA/pBBR1MCS-3-welA was further identified by colony PCR using the specific primers welAinFor/welAinRev for partial welA gene. The wild type containing the empty vector pBBR1MCS-3 named as WT/pBBR1MCS-3 was constructed through triparental conjugal mating using the method as described in our previous work (Li et al., 2021).

\footnotetext{
${ }^{4}$ https://npsa-prabi.ibcp.fr/cgi-bin/npsa_automat.pl?page=/NPSA/npsa_sopma. html

${ }^{5} \mathrm{http} / / /$ smart.embl-heidelberg.de/
}

\section{Measurement of Cell Growth, Broth Viscosity, Exopolysaccharide Production of Different Sphingomonas sp. WG Strains}

To elucidate the possible role of WelA in the EPS production, four different strains, wild-type of Sphingomonas sp. WG (WT), $\triangle$ welA, $\triangle$ welA/pBBR1MCS-3-welA and WT/pBBR1MCS-3 were cultivated in $50 \mathrm{~mL}$ fermentation medium in $250 \mathrm{~mL}$ shake flasks at $32.5^{\circ} \mathrm{C}, 200 \mathrm{rpm}$ for different time. The fermentation parameters such as the biomass, the WL gum production, and the fermentation broth viscosity were measured as previously described ( $\mathrm{Li}$ et al., 2018). The specific growth rate $\mu$ and time for the bacterial population to double (tD) were calculated according to the growth curve. The EPS production was determined with the phenol-sulfuric acid colorimetric method described previously using glucose as the standard (Zhou et al., 2017). The viscosity of fermentation broth was measured on a Brookfield Viscometer DV-III equipped with an ultra-low adaptor (Brookfield Engineering Laboratories) using the rotor spindle LV3 at $5 \mathrm{rpm}$ at $25^{\circ} \mathrm{C}$.

\section{Motility Assay of Different Strains in Semisolid Agar}

The WT, $\triangle$ welA, and $\triangle$ welA/pBBR1MCS-3-welA strains were cultured in the $\mathrm{LB}$ medium at $30^{\circ} \mathrm{C}$ until $\mathrm{OD}_{600}$ was 0.6 . The motility was tested on SIM media or fermentation medium supplemented with $0.3 \%$ (for swimming motility) and $0.5 \%$ (for swarming motility) of agar where $5 \mu \mathrm{L}$ of bacterial suspension was dropped in each plate center and inoculated for $72 \mathrm{~h}$ (for swimming motility) and for $168 \mathrm{~h}$ (for swarming motility) at $28^{\circ} \mathrm{C}$. The radius of circles formed by bacterial migration was measured to express the motility.

\section{RNA Extraction, cDNA Library Construction, Illumina Deep Sequencing, and Data Analysis}

Cells of WT and $\triangle w e l A$ at the exponential phase (about $16 \mathrm{~h}$ of fermentation) were collected and harvested by centrifugation, respectively. Total RNA was extracted using RNAiso Plus (TaKaRa Biotechnology Company, China), and RNA integrity was assessed by an Agilent 2100 Bioanalyzer system (Agilent Technologies, CA, United States). RNA with a RIN value higher than 8.0 was chosen to produce a transcriptome library. According to the manufacturer's instructions, cDNA libraries were generated using a NEBNext Ultra Directional RNA library prep kit for Illumina (NEB, United States). The cDNA library was sequenced on an Illumina sequencing platform (Illumina HiSeq ${ }^{\mathrm{TM}} 4000$ platform) by the Shanghai Personal Biotechnology Co., Ltd. Library quality was assessed on an Agilent Bioanalyzer 2100 system.

Raw data (raw reads) in fastq format were filtered by removing low-quality reads, reads containing adapters, and ploy- $\mathrm{N}$ to obtain high-quality clean data used in all downstream analyses. The high-quality clean reads were mapped to the reference 
genome of Sphingomonas sp. WG (GenBank no. LNOS00000000) using Bowtie2-2.2.3 (Langmead and Salzberg, 2012). The level of gene expression was estimated by FPKM (Fragments Per Kilo bases per Million fragment). Differential expression analysis of the two groups (WT as the control and $\triangle$ welA as the experiment group) was performed using the DESeq (version 1.18.0) (Anders and Huber, 2010). Genes with a minimal twofold difference in expression (| log2 fold change $(\mathrm{FC}) \mid \geq 1$ ) and $p$-value $<0.05$ were identified as differentially expressed genes (DEGs). Thus, the number of up-regulated and down-regulated genes was calculated based on the $\log _{2} \mathrm{FC}$ value.

DEGs with similar expression patterns were clustered by the Pheatmap R package using the Complete Linkage method. Furthermore, functional enrichment analysis was performed by Gene Ontology (GO) and KEGG enrichment analysis. For GO enrichment analysis, each DEG was mapped to GO terms in the Gene Ontology database ${ }^{6}$ by topGO (Alexa et al., 2006). The DEGs were also mapped to the KEGG orthology terms in the KEGG pathway database ${ }^{7}$ using the KOBAS software (Bu et al., 2021).

\section{Experimental Validation of Gene Expression by Quantitative Real-Time PCR (qRT-PCR)}

The expressional levels of 10 typical DEGs identified by RNA-Seq were examined using qRT-PCR. Total RNA of WT and $\triangle$ welA cultured for $16 \mathrm{~h}$ were reverse-transcribed by a PrimeScript $^{\circledR}$ RT Reagent Kit with gDNA Eraser (Perfect Real Time) (TaKaRa, Dalian, China) according to the manufacturer's instructions. qRT-PCR was performed using the primers listed in Supplementary Table 2 with a TB Green ${ }^{\circledR}$ Premix Ex Taq ${ }^{\mathrm{TM}}$ II (Tli RNaseH Plus) kit (TaKaRa, Dalian, China) on ABI 7500 instrument (Applied Biosystems). The qRT-PCR reaction system and conditions were the same as described in our previous work (Li et al., 2018). The relative expression of each gene was calculated according to the $2^{-\Delta \Delta} \mathrm{Ct}$ formula, and the $16 \mathrm{~S}$ rRNA gene served as an internal standard to standardize results. The expression of each gene was repeatedly detected at least three times. The data are expressed as arithmetic means \pm the standard deviation.

\section{Quantitation of Intracellular c-di-GMP by HPLC-MS/MS}

Firstly, WT, $\triangle$ welA, and $\triangle$ welA/pBBR1MCS-welA were cultured in fermentation media for about $12 \mathrm{~h}$ and harvested by centrifugation, respectively. Secondly, nucleotide extraction was carried out as previously reported (Newell et al., 2011) and was resuspended in $500 \mu \mathrm{L}$ water under vigorous vortexing. Finally, the concentration of c-di-GMP was analyzed by reversed phasecoupled HPLC-MS/MS on a Waters Xevo TQ-S Triple Quad Mass Spectrometer using the method reported by Spangler et al. (2010).

\footnotetext{
${ }^{6} \mathrm{http}: / /$ www.geneontology.org/

${ }^{7}$ http://www.kegg.jp/kegg/kegg1.html
}

Heterologous expression of possible diguanylate phosphodiesterase encoded by Gene 1094 in E. coli BL21 (DE3) and its activity assay.

Firstly, the recombinant expression plasmid was constructed. the ORF of Gene 1094 was amplified by PCR using the Sphingomonas sp. WG genomic DNA as the template and gene1094exFor/gene1094exRev containing BamHI and HindIII recognition sties as primers (Supplementary Table 1). Sequentially, PCR product was gel-purified, digested by BamHI and HindIII and ligated into the linearized pET32a ${ }^{(+)}$. The recombinant plasmid pET32a-1094 was confirmed by PCR using gene1094exFor/gene1094exRev as primers and DNA sequencing. Secondly, the recombinant plasmid was transformed into the E. coli BL21 (DE3) host to obtain the expression strain. Thirdly, the protein expression was induced by $0.8 \mathrm{mM}$ isopropyl-Dthiogalactopyranoside (IPTG) at $28^{\circ} \mathrm{C}$ for $16 \mathrm{~h}$ in accordance with the pET system manual (Novagen, Germany). The cells untreated with IPTG was used as the control group. Then the crude enzyme samples were obtained by ultrasonic disruption of the cells, centrifugation and ultrafiltration. The samples were analyzed by sodium dodecyl sulfate-polyacrylamide gel electrophoresis (SDS-PAGE) to confirm the expression of the target protein.

Finally, the hydrolysis reaction of c-di-GMP was carried out in the mixture as follows: $50 \mathrm{mM}$ Tris- $\mathrm{HCl}$ buffer $(\mathrm{pH} 9.35)$ containing $50 \mathrm{mM} \mathrm{NaCl}, 5 \mathrm{mM} \mathrm{MgCl}_{2}$ and $0.5 \mathrm{mM}$ EDTA, $100 \mu \mathrm{M}$ c-di-GMP as the substrate and the crude enzyme. The reaction was performed at $37^{\circ} \mathrm{C}$ for $2 \mathrm{~h}$ and was terminated by heating the samples at $100^{\circ} \mathrm{C}$ for $3 \mathrm{~min}$. The precipitated proteins were removed by centrifugation at $16,000 \times g$ and the obtained supernatant was filtered using a $0.22 \mu \mathrm{m}$ membrane and analyzed by an HPLC instrument (Agilent 1260 Infinity II) equipped with a ZORBAX SB-C18 column $(15 \times 4.6 \mathrm{~mm})$ and detected at $254 \mathrm{~nm}$. The mobile phase A was $100 \mathrm{mM} \mathrm{KH}{ }_{2} \mathrm{PO}_{4}$ containing $4 \mathrm{mM}$ tetrabutylammonium hydrogen sulfate, and mobile phase B contained $75 \%$ phase A and $25 \%$ methanol. The phase $\mathrm{B}$ content in mobile phase was gradient from 0 to $30 \%$ from 2.5 to $5 \mathrm{~min}$; 30 to $60 \%$ from 5 to $10 \mathrm{~min}$; 60 to $100 \%$ from 10 to $14 \mathrm{~min}$ and at $100 \%$ for $7 \mathrm{~min}$, then from 100 to $50 \%$ from 21 to $22 \mathrm{~min}$ and finally to 0 at $23 \mathrm{~min}$. c-di-GMP was used as the standard. The enzyme activity $(1 \mathrm{U})$ was defined as the amounts of enzyme required to hydrolyze $1 \mu \mathrm{M}$ c-di-GMP per minute in $1 \mathrm{ml}$ reaction mixture.

\section{RESULTS}

\section{Bioinformatical Analysis of WelA}

One putative hybrid sensor histidine kinase (HK)/response regulator (RR) encoding gene welA was annotated in the genome of Sphingomonas sp. WG ( $\mathrm{Li}$ et al., 2016a). The length of the open reading frame of welA was $2,385 \mathrm{bp}$, and therefore, the deduced protein WelA contained 794 amino acids. According to the predicted results of ProtParam, the calculated molecular mass and $\mathrm{pI}$ of WelA were $84.5 \mathrm{kDa}$ and 5.14 , respectively. The secondary structure prediction results by SOMPA showed the percentages of $\alpha$-helix, extended strand, $\beta$ turn, and random coil 
predicted were about $42.57,18.14,6.30$, and 33.00. Structural domain analysis by SMART showed that this protein contains two PAS domains (residue 76-145 and residue 293-358), a HisKA domain (residue 418-484), a HATPase_c domain (residue 525653), and a REC domain (residue 676-787), suggesting that this protein had both HK and RR domains and might be a hybrid sensor HK/RR. Besides, it also had two transmembrane regions (residue 15-34 and 41-63), indicating that it might be associated with the cell membrane.

Subsequently, proteins homologous to WelA were retrieved from the Swissprot database by the BLASTp program. A phylogenetic tree was constructed by ClustalX multiple sequence alignment program version 2.0 (Larkin et al., 2007) and MEGA 7 program (Kumar et al., 2016; Figure 1A). WelA from Sphingomonas sp. WG showed $50.12 \%$ identity with the hybrid sensor HK CckA (Genbank no. P0DOA0.1) (Supplementary Figure 1) which initiated the conserved CckA-ChpT-CtrACpdR regulatory system controlling cell division, replication, and virulence of Brucella abortus (Willett et al., 2015). It also showed similarity to other sensor kinases such as DivL and FixL in varying degrees.

\section{The Effect of WelA on WL Gum Production}

To investigate if WelA is involved in the regulation of WL gum biosynthesis, welA was deleted from the genome of Sphingomonas sp. WG by double homologous recombination using the method reported in our previous work (Li et al., 2021). The mutant strain $\triangle$ welA was verified by PCR with primers welAinFor and welAinRev (Figure 1B), and the specific product about $530 \mathrm{bp}$ was lost. Besides, the welA coding sequence was introduced into $\triangle$ welA to obtain the complemented strain $\triangle$ welA/pBBR1MCS3-welA, which was also elucidated by amplifying the partial welA coding sequence using welAinFor and welAinRev as primers (Figure 1C).

Subsequently, the fermentation of the WT, $\Delta$ welA, and $\Delta$ welA/pBBR1MCS-3-welA was performed (Figure 2). As the fermentation proceeded, the WT strain and the $\Delta$ welA strain entered the log phase or exponential phase very quickly (within $6 \mathrm{~h}$ ), and their biomass increased significantly. Compared with WT strain, the deletion of welA did not reduce the cell growth rate, in contrast, it led to the enhanced growth rate. The $\mu_{\max }$ for WT strain and $\Delta$ welA in the log phase was about 0.17 and $0.25 \mathrm{~h}^{-1}$, respectively. $\triangle$ welA/pBBR1MCS-3welA showed a different growth curve in which it had a long lag phase for about $21 \mathrm{~h}$ and then entered the log phase with a $\mu$ about $0.12 \mathrm{~h}^{-1}$. The $\mathrm{tD}$ time for WT, $\Delta$ welA, and $\Delta$ welA/pBBR1MCS-3-welA was $4.26,3.23$, and $5.85 \mathrm{~h}$, respectively. The slow specific growth rate of the complementary strain might be related to the replication burden of the plasmid pBBR1MCS-3-welA or the dose-dependent effect of the welA overexpression. Therefore, the growth curve of the other strain WT/pBBR1MCS-3 was determined. The empty vector had a very slight impact on the growth of the bacteria. Therefore, the slow growth rate of the complemented strain might be caused by a dose-dependent effect of the welA overexpression. Due to the influence of the metabolites such as organic acids, the $\mathrm{pH}$ of the fermentation broth changed. The $\mathrm{pH}$ value of WT was decreased and was the lowest among the three strains. The $\mathrm{pH}$ of the $\Delta$ welA/pBBR1MCS-3-welA was the highest, which might be related to its lowest biomass. Furthermore, the viscosity of the fermentation broth was detected as the accumulation of the EPS will enhance the viscosity. The viscosity of $\Delta$ welA fermentation broth was only $0.6 \%$ of WT, while the broth viscosity of the complemented strain could restore to $40 \%$ and $44 \%$ when cultured for 72 and 84 h, respectively. Similar to viscosity, welA regulated the $\mathrm{WL}$ gum biosynthesis, and the deletion of welA resulted in a remarkable decrease in WL gum production. The WL gum yield of $\Delta$ welA was only $44 \%$ and $49 \%$ of WT when cultured for 72 and $84 \mathrm{~h}$. The gene complementation could restore the WL gum production to $73 \%$ and $74 \%$ of WT at 72 and $84 \mathrm{~h}$. All these phenomena suggested that WelA plays important regulatory role in the WL gum biosynthesis process.

\section{Transcriptome Analysis of WT Strain and $\Delta$ welA Mutant Strain}

Transcriptome analysis of WT (the control group) and $\Delta$ welA (the experiment group) was conducted to understand the genes differentially regulated by WelA. An average of 32,410,509 and $36,273,418$ raw sequencing reads in the control group and experimental group was generated by Illumina sequencing, respectively. After the trimming process, about $93 \%$ and $90 \%$ of the libraries remained clean reads, respectively (Supplementary Table 3). The correlation of gene expression level between samples was checked to test the sequencing reliability of the experiment and the rationality of sample selection, and the results were shown in Supplementary Figure 2. The square of Pearson correlation coefficient $\left(R^{2}\right)$ between biological repeats was higher than 0.92 and suggested that the difference between individuals was very small and the sequence results were credible. Moreover, the six samples could be clustered into two groups, the WT and $\Delta$ welA group, which indicated that the deletion of welA resulted in a significant change of gene expression (Supplementary Figure 2). Therefore, differential expression analysis was performed. A total of 386 DEGs with 187 upregulated genes and 199 down-regulated genes were detected (Supplementary Figure 3). To validate the accuracy of RNAseq results, the expression of 10 typical DEGs that involved in signaling transduction pathway, sugar metabolism and WL gum biosynthesis, and c-di-GMP biosynthesis or hydrolysis was further confirmed by qRT-PCR. The results were shown in Figure 3. According to correlation analysis, the expression results of qRT-PCR were strongly positively consistent with those from RNA-Seq $\left(R^{2}=0.7642\right)$, which suggested that the data from the transcriptome were reliable.

\section{Gene Ontology (GO) and Kyoto Encyclopedia of Genes and Genomes (KEGG) Enrichment Analysis}

GO enrichment analysis was performed to gain the information of DEGs functional categories. The results (Figure 4) showed that the "bacterial-type flagellum (GO:0009288)" and "cell 
A

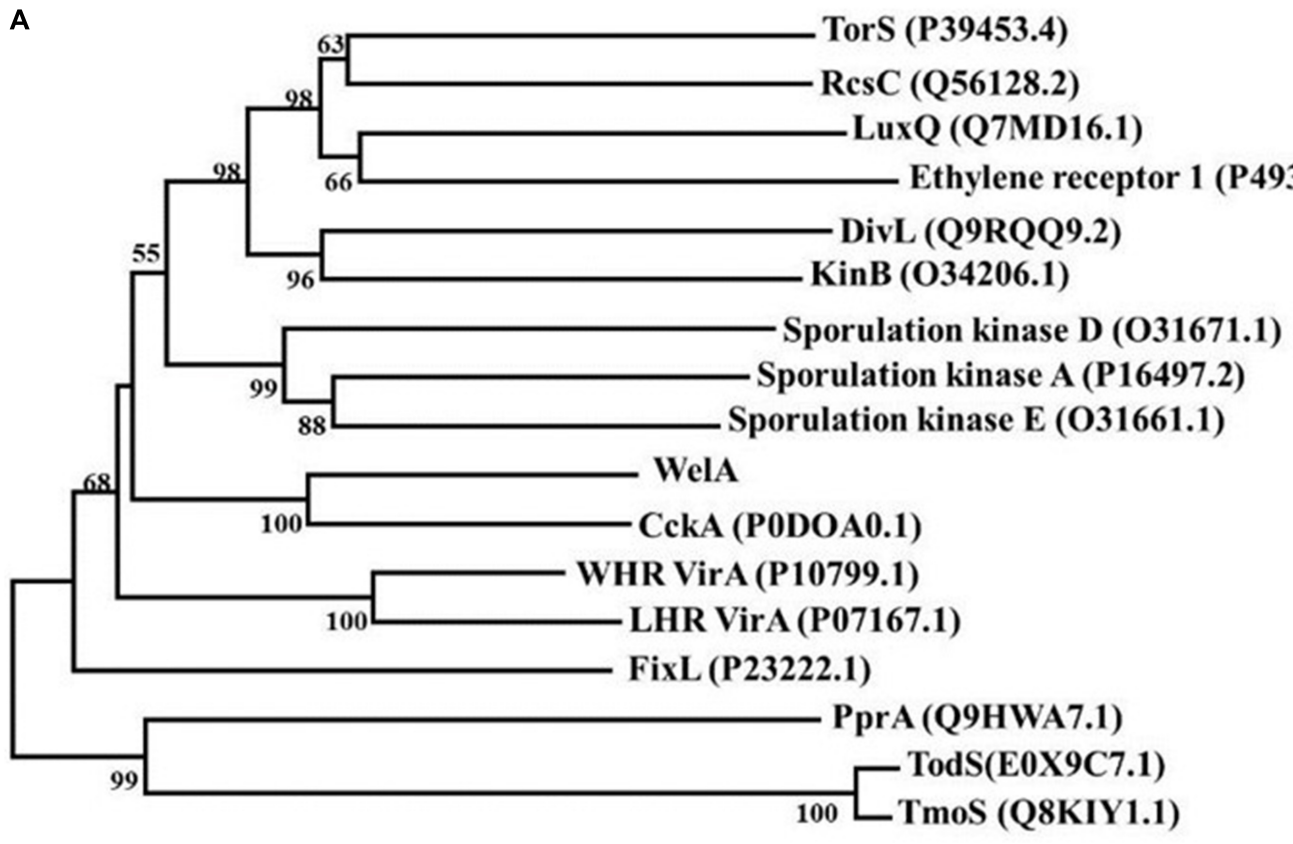

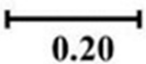

B

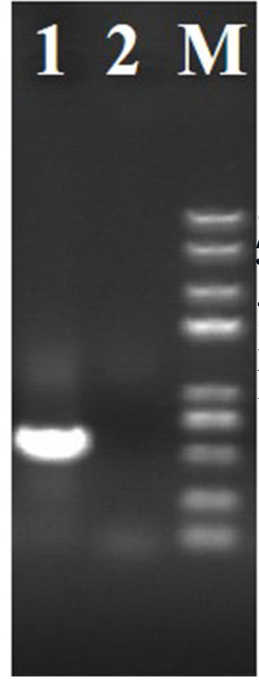

C

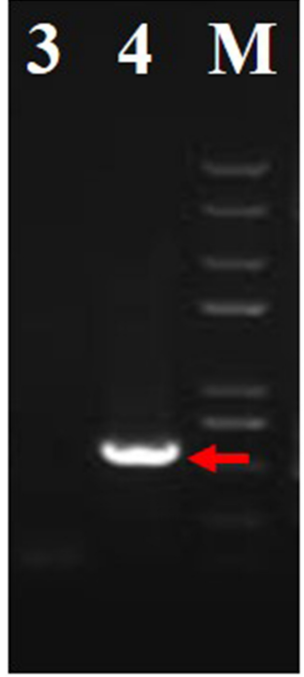

8000 bp 5000 bp 3000 bp 2000 bp 1000 bp 750 bp 500 bp 250 bp $100 \mathrm{bp}$

FIGURE 1 | The phylogenetic analysis of WelA and verification of welA knock-out and complemented strain. (A) The phylogenetic tree of WelA based on the amino acid sequences. Sequence alignment was conducted by ClustalX2 software, and the tree was generated by MEGA 7 software using the neighbor-joining method. Bootstrap values, expressed as the percentages of 1,000 replications, are given at the branching points when they are higher than 50 . Scale bar, 0.20 amino acid substitutions/site. (B) Identification of $\Delta$ welA mutant by PCR using primers welainfor/welainrev for partial welA encoding sequence. The PCR templates of different lanes were as follows: Lane 1: WT strain, Lane 2: $\Delta$ welA mutant. (C) Identification of the complemented strain by PCR using primers welAinFor and welAinRev for partial welA coding sequence. The PCR templates of different lanes were as follows: Lane 3: $\Delta$ welA mutant as negative control, Lane 4: complemented strain, M: D8000 DNA marker with the bands at 8,000, 5,000, 3,000, 2,000, 1,000, 750, 500, 250, and 100 bp. The right band was indicated by the arrow.

projection (GO:0042995)" were the top GO terms in the "cellular component" group; The "methyltransferase activity (GO:0008168)" and "transferase activity, transferring one-carbon groups (GO:0016741)" were significantly enriched in "molecular function" group. The "secretion (GO:0046903)," "secretion by cell (GO:0032940)," "protein secretion (GO:0009306)," and "peptide secretion (GO:0002790)" were significantly more clustered in the "biological process category." Besides, the DEGs were also mapped to different KEGG categories, including cellular processes, environmental information processing, genetic information processing, human diseases, and metabolism. The top 20 enriched pathways were also shown in Figure 4. The 

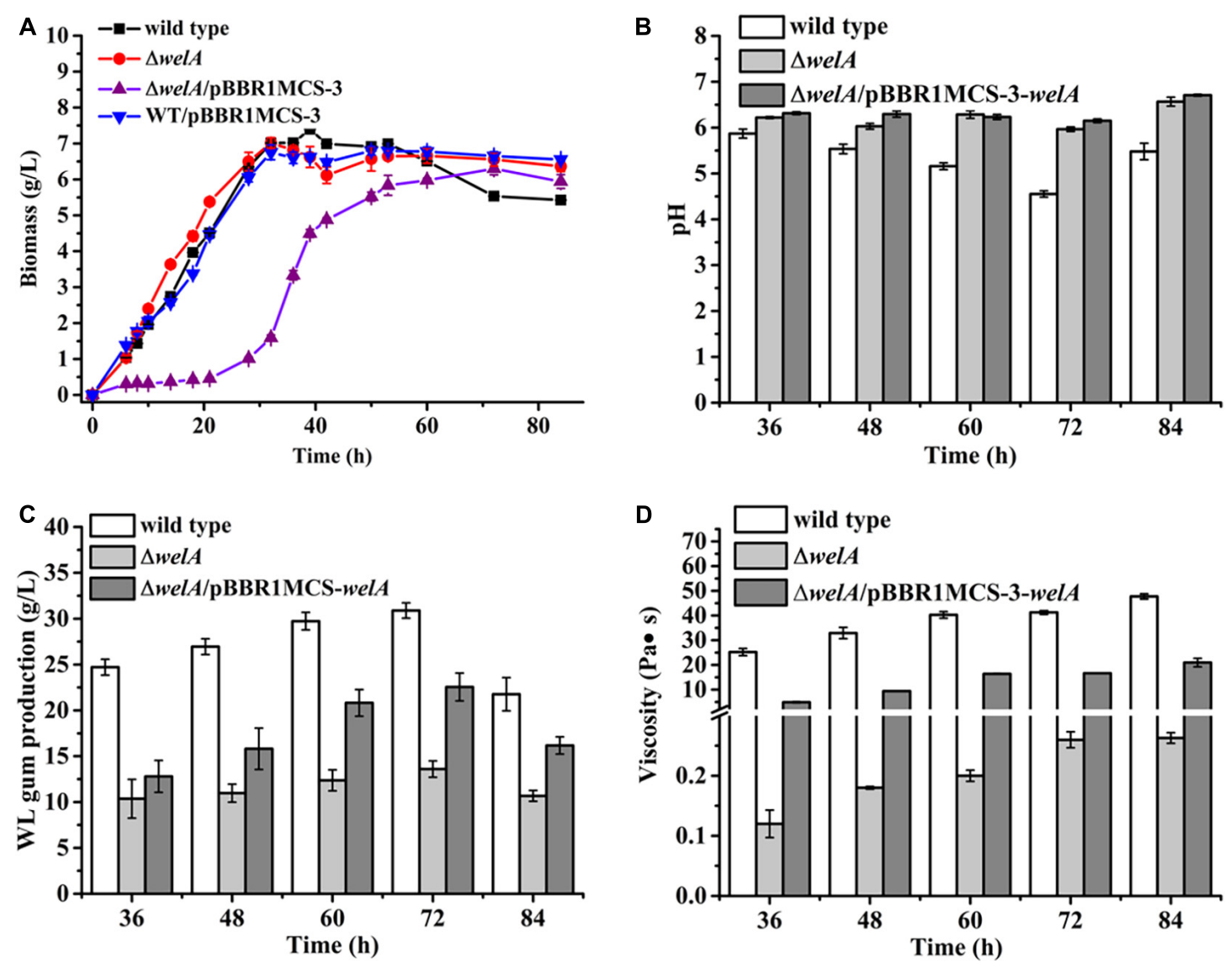

FIGURE 2 | The fermentation results of WT, $\Delta$ welA mutant, and complemented strain $\triangle$ welA/pBBR1MCS-3-welA. (A) Comparison of biomass. (B) Comparison of $\mathrm{pH}$. (C) Comparison of broth viscosity. (D) Comparison of WL gum production.

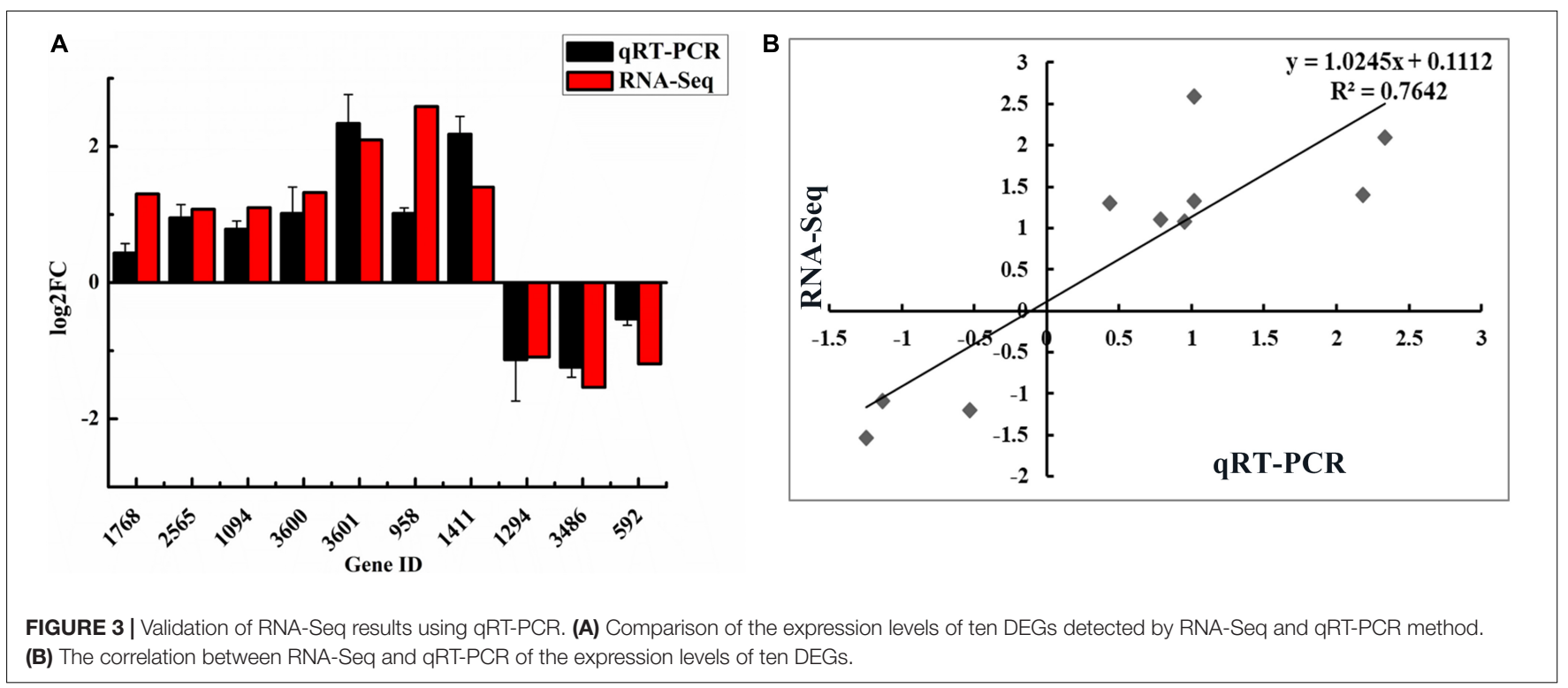

most DEGs were enriched in "Flagellar assembly (ko02040)," "Porphyrin and chlorophyll metabolism (ko00860)," "Valine, leucine, and isoleucine biosynthesis (ko 00290)," "C5-Branched dibasic acid metabolism (ko00660)." Since we focused on the role of WelA on the biosynthesis of WL gum, the regulation pathway of WelA was mainly analyzed in the following step. 

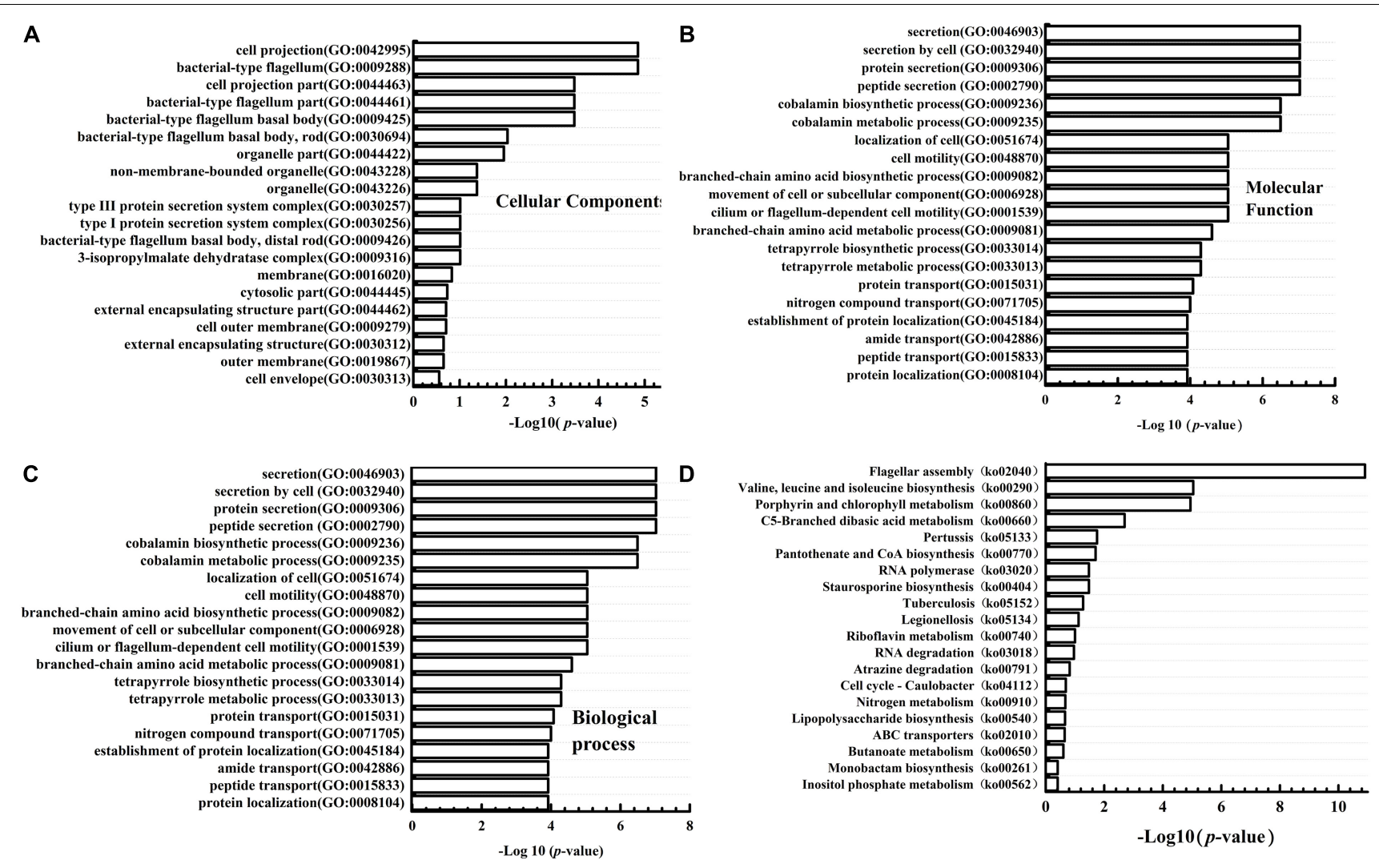

FIGURE 4 | Gene ontology (GO) classification and Kyoto Encyclopedia of Genes and Genomes (KEGG) pathway enrichment analysis of the differentially expressed genes in $\triangle$ welA. (A) The DEGs categorized based on Cell component; (B) the DEGs categorized based on Molecular function; (C) the DEGs categorized based on biological process. (D) KEGG pathway enrichment analysis of DEGs.

\section{DEGs in Two-Component Signal Transduction Systems}

WelA was mapped to the two-component signal transduction systems (ko02020) due to its identity to CckA, and its deletion resulted in the up-regulated expression of chpT (Gene 3285) and $\operatorname{ctr} A$ (Gene 1411) (Table 1). It has been reported that CtrA is an active transcriptional regulator controlling genes involved in cell cycle progression and flagellar motility in Caulobacter crescentus (Laub et al., 2000). Besides CtrA, CpdR (Gene 1015) is another RR that can also be phosphorylated by ChpT. Normally, CpdR inhibits CtrA, and this inhibition effect could be relieved by phosphorylation. However, the expression level of CpdR was not significantly changed after welA deletion. In general, genes controlled by CtrA are enriched in certain functional categories, i.e., cell motility, signal transduction, and cell wall/membrane envelope biogenesis (Brilli et al., 2010). In C. crescentus, the architecture of the CtrA promoters has been analyzed, and two classes of CtrA binding motifs: a full site represented by the sequence TTAA $\left(\mathrm{N}_{7}\right)$ TTAA, and a half site (TTAA) were revealed (Zhou et al., 2015). It has been reported that the expression of the fla2 genes involved in the biosynthesis of polar flagella of Rhodobacter sphaeroides is dependent on the two-component system, including CckA-ChpT-CtrA (Vega-Baray et al., 2015). Based on the motif sequence scanning, CtrA might regulate the expression of many genes. The expression of two special genes involved in c-di-GMP hydrolysis and flagellar assembly was upregulated by CtrA. The first gene was Gene1094 encoding the diguanylate phosphodiesterase that might be related to hydrolysis of c-di-GMP. The heterologous expression of Gene 1094 in E. coli BL21 (DE3) showed that the crude enzyme had weak activity to catalyze the hydrolysis of c-di-GMP (Supplementary Figure 4). Its highly expression level indicated that c-di-GMP level might decreased and was involved in the regulation of WL gum biosynthesis. Therefore, the intracellular c-di-GMP was extracted and determined. As expected, the intracellular c-di-GMP concentration was significantly decreased in $\triangle$ welA (Supplementary Figure 5). Generally, reduced c-di-GMP level might increase flagellar motility and decrease the biofilm formation (Floyd et al., 2020). The second one is FlgN (Gene 283), which is the flagellar synthesis and assembly chaperone, and therefore, the motility might be changed.

Besides the CckA-ChpT-CtrA pathway, the other three genes were mapped to the two-component signal transduction systems (ko02020), including the up-regulated DctP (Gene1533) in the C4-Dicarboxyate transport, the downregulated CheR (Gene 3280) in flageller assembly, and Cyt C (Gene 371) in electron transfer system in Oxidative phosphorylation process. 


\section{Genes Involved in WL Gum Biosynthesis}

Many genes were involved in the biosynthesis of WL gum. However, most genes related to the biosynthesis of nucleotidesugar precursors such as $p g m$, ugp, ugd, and $\operatorname{rm} A$ to $r m l D$, the tetrasaccharide repeating unit assembly such as $w e l B$, welK, welL, and welQ, polymerization of the assembled repeating units such as welS, welG, welE, welC, etc., were not identified as DEGs (Table 1). The expressional level of gene encoding

TABLE 1 | The expression level of genes in two-component system and in WL gum biosynthesis.

Gene ID $\quad \begin{gathered}\text { Gene product } \\ \text { description }\end{gathered} \log _{2} \mathrm{FC} \quad p$-value

\section{DEGs in two-component system}

\begin{tabular}{|c|c|c|c|}
\hline Gene3285 & $\begin{array}{c}\text { Histidine } \\
\text { phosphotransferase ChpT }\end{array}$ & 1.12 & 0.0036 \\
\hline Gene1411 & $\begin{array}{l}\text { Cell cycle transcriptional } \\
\text { regulator CtrA }\end{array}$ & 1.40 & 1.86E-09 \\
\hline Gene1015 & $\begin{array}{l}\text { Two-component system } \\
\text { cell cycle RR CpdR }\end{array}$ & 0.67 & 0.0087 \\
\hline Gene1094 & $\begin{array}{c}\text { Diguanylate } \\
\text { phosphodiesterase }\end{array}$ & 1.10 & 0.026 \\
\hline Gene283 & Flagellar protein FlgN & 1.09 & 0.0053 \\
\hline Gene1533 & $\begin{array}{c}\text { C4-dicarboxylate transport } \\
\text { protein DctA }\end{array}$ & 1.88 & $2.55 \mathrm{E}-05$ \\
\hline Gene 3280 & Chemotaxis protein CheR & -1.01 & 0.0023 \\
\hline Gene 371 & Cytochrome C & -1.44 & 0.025 \\
\hline \multicolumn{4}{|c|}{ Genes in WL gum Biosynthesis } \\
\hline Gene ID & Gene product & $\log _{2} \mathrm{FC}$ & $p$-value \\
\hline Gene3585 & WelG & 0.21 & 0.31 \\
\hline Gene3586 & Wels & 0.17 & 0.50 \\
\hline Gene3587 & WelR & 0.26 & 0.23 \\
\hline Gene3588 & WelQ & 0.34 & 0.16 \\
\hline Gene3589 & Well & 0.08 & 0.74 \\
\hline Gene3590 & Welk & -0.11 & 0.58 \\
\hline Gene3591 & Well & -0.41 & 0.075 \\
\hline Gene3592 & WelJ & 0.23 & 0.33 \\
\hline Gene3593 & Hypothetical protein & 0.48 & 0.17 \\
\hline Gene3594 & WelF & 0.33 & 0.17 \\
\hline Gene3595 & WelD & 0.56 & 0.021 \\
\hline Gene3596 & WelC & 0.47 & 0.036 \\
\hline Gene3597 & WelE & 0.45 & 0.063 \\
\hline Gene3598 & WelM & 0.26 & 0.25 \\
\hline Gene3599 & WelN & -0.64 & 0.0084 \\
\hline Gene3600 & AtrD & 1.32 & 0.00033 \\
\hline Gene3601 & AtrB & 2.09 & 1.1E-05 \\
\hline Gene3602 & Hypothetical protein & -0.25 & 0.30 \\
\hline Gene3603 & WelB & 0.67 & 0.0031 \\
\hline Gene3604 & RmlA & 0.12 & 0.64 \\
\hline Gene3605 & RmlC & 0.38 & 0.24 \\
\hline Gene3606 & RmlB & -0.08 & 0.87 \\
\hline Gene3607 & RmlD & -0.74 & 0.0016 \\
\hline Gene 896 & PGM & 0.65 & 0.094 \\
\hline Gene3404 & UGP & 0.53 & 0.026 \\
\hline Gene3147 & UGD & 0.63 & 0.031 \\
\hline Gene592 & PMM & -1.19 & 5.6E-06 \\
\hline
\end{tabular}

PMM (Gene 592) catalyzing the synthesis of $\alpha$-D-mannose 1phosphate, the precursor of GDP-mannose, was down-regulated (the $\log 2 \mathrm{FC}$ was -1.19). Besides, two genes (Gene 3600 and 3601) encoding $\mathrm{AtrD}$ and $\mathrm{AtrB}$ in the wel cluster were identified as up-regulated DEGs.

\section{Regulation of Flagella Synthesis and Motility of WelA}

According to the GO enrichment results (Figure 4), the DEGs under the category of biological process related to processes of flagellum-dependent cell motility (GO:0001539) and under the categories of the cellular component involved in bacterialtype flagellum (GO:0009288) were highly expressed. KEGG pathway analysis revealed that 14 DEGs were involved in flagellar biosynthesis, including 12 highly and 2 lowly expressed genes. Taken together, WelA might play a regulatory role in flagellar biosynthesis of Sphingomonas sp. WG. Therefore, the organization of the genes for flagellar biosynthesis was analyzed. Most of the genes were clustered together in the smaller cluster I and the larger cluster II within the chromosome, as shown in Figure 5 and Supplementary Table 4. Most of the highly expressed genes in $\triangle$ welA encode essential components for flagellar biosynthesis, including flagellin, flagellar basal body protein, flagellar hook-associated protein, and export apparatus for flagellar components. Furthermore, three genes that encode transcriptional regulatory proteins FlbD, FlaEY, and FlaF were also highly expressed. However, some genes in the chemosensory system that also influence motility, such as CheR, McpA, and twitching motility protein PilT, were down-regulated.

Therefore, it is inferred that the flagellar formation and the motility of the mutant strain might change. Therefore, the swimming and swarming motilities of WT and welA mutant strain were measured. $\Delta$ welA showed higher swimming motility than WT on SIM medium (Figure 5C); however, when cultured on solid fermentation medium, its swimming motility was much lower (Figure 5D). Its swarming motility independent of the presence of a flagellum was lost on both media (Figure 5E).

\section{DISCUSSION}

In prokaryotes, two-component signal transduction systems are the predominant means to respond to a wide range of external stimuli such as antibiotics and quorum-sensing signals. Normally, each two-component signal transduction system consists of a sensor HK protein that can be autophosphorylated and a RR protein with the N-terminal receiver domain (REC) receiving the phosphoryl group required for activation of the regulatory domain. Through the signal transduction, many physiological changes will be triggered by changing gene expression programs, altering swimming behavior etc. (Skerker et al., 2005; Mattos-Graner and Duncan, 2017). As a hybrid sensor HK/RR, besides HisKA and REC domain, WelA also contained two common conserved signal input PAS domains suggesting that the protein has a "sensor" capacity (Christensen and Serbus, 2015). Therefore, WelA might play important roles in WL gum biosynthesis and other cellular physiology. As expected, 


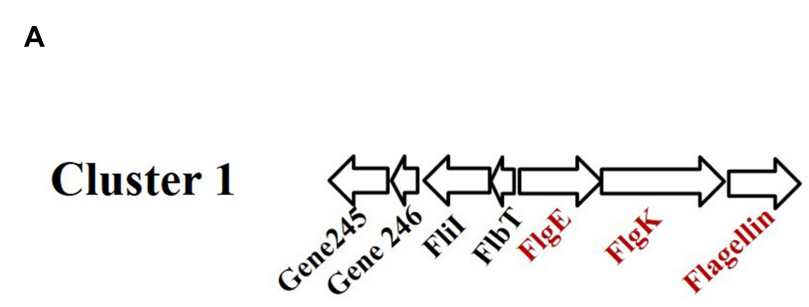

\section{Cluster 2}
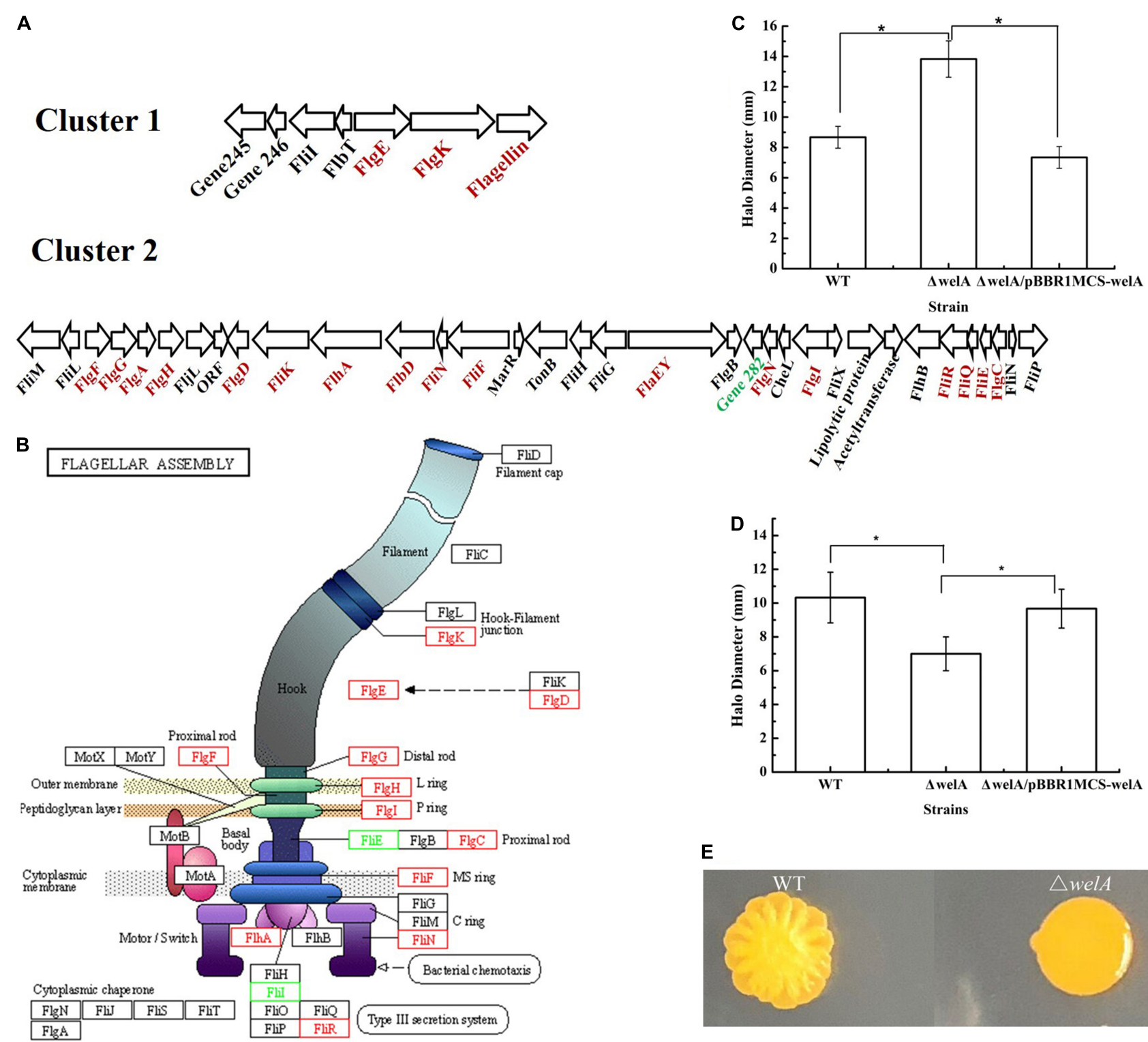

E

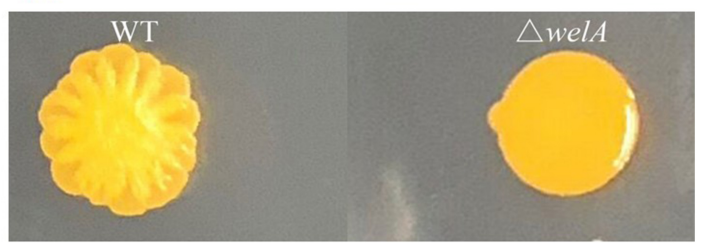

FIGURE 5 | The expressional levels of genes involved in flagellar assembly and effects on motility. (A) Cluster I and II involved in flagellar assembly. Highly expressed genes are red and lowly expressed genes are green. (B) The Schematic diagram of the differentially expressed genes involved in flagellar assembly (KEGG pathway k002040). Highly expressed genes are red and lowly expressed genes are green. (C) Swimming motility on SIM medium. (D) Swimming motility on solid fermentation medium. (E) Swarming motility. ${ }^{\star}$ means $p<0.05$.

WelA affected the WL gum production and fermentation broth viscosity through gene deletion manipulation. Through KEGG enrichment analysis, WelA was mapped to CckA in the branched CckA-ChpT-CtrA phosphorelay pathway. The deletion of welA resulted in the hyperactive expression of ChpT and CtrA. Similarly, the deletion of cckA in Rhodobacter capsulatus resulted in increased expression of ChpT and CtrA (Koppenhofer and Lang, 2020). Besides CckA, ChpT might also receive signals from other kinase and transduce them to CtrA (Koppenhofer and Lang, 2020). The activity of CtrA was also higher in Sphingomonas melonis $\triangle c c k A$ than that of WT strain, which might be due to the absence of CckA phosphatase activity (Francez-Charlot et al., 2015). CtrA is a master regulator of motility and regulates EPS synthesis and cyclic-di-GMP signaling in S. melonis (Francez-Charlot et al., 2015). Thus, the more expressed ChpT and CtrA might influence many cellular metabolisms. One carbon metabolism was critical for DNA methylation and DNA synthesis (Lee et al., 2009) because it plays an important role in the formation of many methyl donors such as S-adenosylmethionine and donors that could be recognized by DNA, RNA, histone, and protein methyltransferases (Lyon et al., 2020). DNA methylation regulated many cellular processes such as protein-protein interactions, DNA replication initiation and gene expression in 
bacteria (Sánchez-Romero and Casadesús, 2020). Therefore, the enriched genes in "transferase activity, transferring one-carbon groups (GO:0016741)" might involve in the gene expression regulation in welA mutant strain.

Firstly, the higher expressional level of genes involved in the flagellar assembly in welA mutant strain was detected. Many genes are required for the bacterial flagellum assembly, such as structural subunits, regulatory proteins, motor proteins, export apparatus for flagellar components, and the chemosensory machinery (Aldridge and Hughes, 2002). In many bacterial species, the expression of the flagellar genes was regulated in a hierarchical pattern in which genes might be classified into III classes and IV classes in some cases. For example, the transcriptional activator FlhD/FlhC, the sigma factor FliA $\left(\delta^{28}\right)$, and its specific anti-sigma factor FlgM regulated the hierarchical expression of flagellar genes in E. coli and Salmonella enterica (Rivera-Osorio et al., 2018). The FlhD and FlhC activated the genes for the hook and basal body assembly and FliA with the help of the sigma factor $\left(\delta^{70}\right)$. When the basal body and the hook are assembled, the exportation of FlgM is occurred, which resulted in the association of FliA with the core RNA polymerase to recognize the promoters of genes to facilitate filament formation and chemotaxis (Maruyama et al., 2015). In several species, the expression of the early flagellar genes is induced by sigma factor RpoN and an activator protein, while the late genes are regulated by FliA and FlgM (Smith and Hoover, 2009; Tsang and Hoover, 2014). In C. crescentus and Sinorhizobium meliloti, the expression of several genes involved in flagellar biosynthesis is directly activated by the transcriptional regulator CtrA (Pini et al., 2015; Rivera-Osorio et al., 2018). However, there is little information about flagellar biosynthesis and motility of Sphingomonads. In Sphingomonas wittichii, the genes involved in the biosynthesis and assembly of flagellar are in three chromosomal regions (Swit_0212-0213, Swit_1260-1293, and Swit_1458) and its biosynthesis might be regulated by a putative Fli-type RNA polymerase sigma28 factor (Swit_1281). Furthermore, three genes encoding pili assembly proteins (Swit_0565, Swit_0615, and Swit_0616) were also characterized (Johnson et al., 2011). Two types (lateral and polar) of flagellar gene sets were found in Sphingomonas sp. strain A1, although it has a single polar flagellum, and the set II clusters contained the regulatory protein-encoding genes $f l h C, f l h D$ and fliA. However, the regulation mechanism was unclear because no common motif for FlhD2C2 binding was found in its genome (Maruyama et al., 2015).

The organization of genes for flagellar biosynthesis in Sphingomonas sp. WG was similar to that of the sphinganproducing Sphingomonas sp. strain S2M10 (Czieborowski et al., 2020). However, it was different from that in Sphingomonas melonis (Francez-Charlot et al., 2015) and Sphingomonas A1 (Maruyama et al., 2015). The commonly regulatory proteinencoding gene such as $f h C$, flhD and fliA was not found in clusters I and II. It seemed that the hierarchical regulation pattern of flagellar biosynthesis in Sphingomonas sp. WG was similar to C. crescentus with the regulatory proteins CtrA, the $\delta^{54}$ transcriptional activator FlbD and its trans-acting regulator FliX, the repressor protein FlbT and post-transcriptional regulator
FlaF, and the possible regulatory protein FlaEY. Genes encoding FlbD, FliX, FlbT, and FlaEY were in the cluster, and those encoding FlaF and CtrA were in other loci of the genome. The expressional level of $f l b T$ and $f l i X$ was not changed significantly. However, ctrA, flbD, flaEY, and flaF were up-regulated. CtrA might regulate the expression of $f l g N$, which encodes the flagellar synthesis and assembly chaperones through the binding motif screening. Besides FlgN, three proteins FlhA, FliQ, and FliR were also up-regulated, which belong to the transmembrane export gate that acts as a proton/protein antiporter in the export machinery. The enhanced expression of FlgN, FlhA, FliQ, and FliR might be beneficial for the export machinery to transport the structural subunits of flagellar from the cytoplasm to the growing flagellar distal end (Minamino et al., 2021). FlbD and FliX tightly controlled the expression of the genes involved in the assembly of LP-ring, distal rod, and hook of the subpolar flagellum, and the extracellular polysaccharide production and biofilm formation in Bradyrhizobium diazoefficiens (Dardis et al., 2021). FlaE and FlaY have been proved to modulate the expression level of the flagellin genes and several genes encoding chemotaxis functions in C. crescentus (Minnich et al., 1988). In Sphingomonas sp. strain S2M10, the mutant of FlaQ, the homolog of FlaEY, was proved to enhance the expression of $f l i C$ and reduce the expression of $f l g I$ and a hypothetical protein (Czieborowski et al., 2020). Therefore, the more expressed master regulators might participate in the regulation of flagellar biogenesis; however, the flagellar regulatory cascade remains unclear.

Moreover, the changes of flagellar were related to the motility of the cells. For most bacteria, flagella are the major organelles responsible for swimming motility and type IV pili (TFP) are responsible for the twitching motility. The swimming motility might be related to the length of flagellum. When the FlaC was knocked out, short flagellum was formed and the swimming motility was decreased (Jung et al., 2021). In our work, we found that the swimming motility of the mutant stain was dependent on the medium, which might be related to the complicated regulation mechanism of flagellar formation. In the other respect, the swarming motility of Pseudomonas aeruginosa is dependent on flagella, TFP and presence of rhamnolipids (Overhage et al., 2007). Therefore, the down-regulated twitching motility protein PilT, an ATPase responsible for TFP, might lead to the decreased swarming motility.

The expressional levels of genes involved in sphingan WL gum production must also be given attention. Most genes in the biosynthesis pathway were not identified as DEGs. Similar results were observed in Sphingomonas sp. ATCC 31555. The expressional level of ten genes in welan gum biosynthesis was not changed between the inorganic nitrogen group, organic nitrogen group, and inorganic-organic combined nitrogen group, although their welan gum production was different ( $\mathrm{Xu}$ et al., 2017). In $\Delta$ welA, only three related genes encoding PMM, AtrB, and AtrD were DEGs. AtrB and AtrD showed similarity to several ABC-type transporter proteins from diverse genera of bacteria. They were mapped as HlyB/CyaB and HlyD/CyaD in the type I secretion system. However, the function of $A \operatorname{trB}$ and $A \operatorname{trD}$ in WL gum biosynthesis was unknown. $\mathrm{ABC}$ transporters are important components of a 


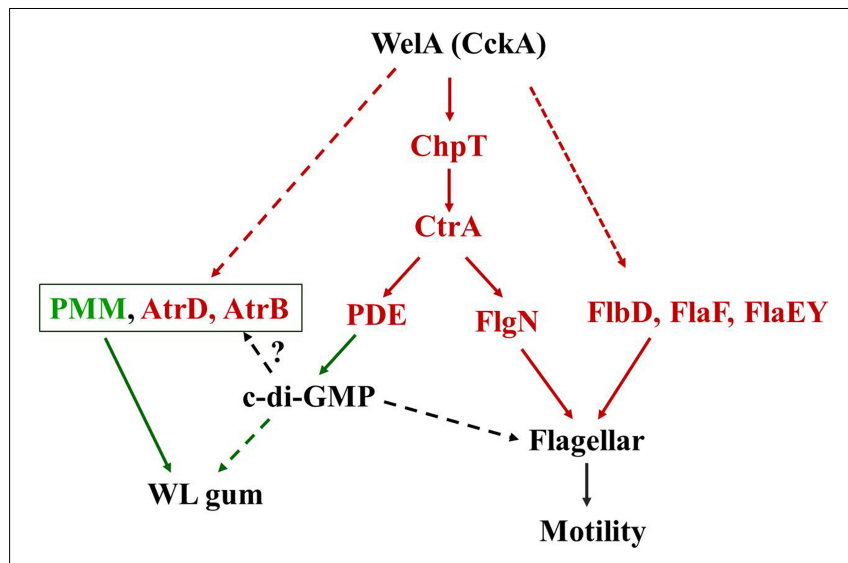

FIGURE 6 | A tentative model for the WelA regulatory circuit to regulate the WL gum biosynthesis and flagellar motility. The solid lines indicate the potential phosphate flow from CckA to CtrA via ChpT or activation of regulatory proteins. The dashed lines indicate the unknown regulatory pathway. Proteins in red color were up-regulated whereas those in green color were down-regulated. The red arrows indicated that the interactions result in activation while the green one indicated the inhibiting interactions.

large class of proteins involved in many biological processes, such as uptake of nutrients and export of proteins, drugs, and capsular polysaccharides in many Gram-negative bacteria (Willis and Whitfield, 2013). The over-expression of AtrB and AtrD resulted in significant enhancement of WL gum production of Sphingomonas sp. WG (Data unpublished). Therefore, the enhanced AtrB and AtrD might be related to the WL gum biosynthesis in an unknown way. Besides, the lower expression of phosphomannomutase encoding gene pmm might lead to less GDP-mannose precursor formation, and thereby less WL gum biosynthesis. Furthermore, the regulation of EPS was at the mRNA level and at the protein level. The over-expression of GelA (the homologous of WelA) enhanced Gellan gum (Lee et al., 2017) and the EPS produced by Sphingobium chungbukense DJ77, and resulted in the up-regulated EPS export protein and one unknown protein and the down-regulated putative glucose dehydrogenase and bifunctional acetyltransferase. As the critical components for biofilm formation or virulence, many extracellular polysaccharides are directly or indirectly activated by the second massager cyclic-di-GMP, which plays important role in the intercellular signal transduction (Schmid et al., 2018). The intracellular concentration of this c-diGMP can be modulated by the synthesis and degradation catalyzed by diguanylate cyclases and specific phosphodiesterases under environmental and physiological cues through complex signal transduction systems. Generally, high concentrations of c-di-GMP promote biofilm formation, including extracellular polysaccharides and other matrix components biosynthesis. c-di-GMP binds to specific effector proteins such as Alg44 with PilZ domain, FimX with the EAL domain, PopA with RxxD motif, and Clp or the responsive riboswitch (GEMM motif) (Boyd and O'Toole, 2012). The expression of one possible phosphodiesterase regulated by CtrA was enhanced. Therefore, the intracellular c-di-GMP was decreased in $\triangle$ welA.
The lower c-di-GMP might involve in the WL gum biosynthesis and lead to the lower WL gum production. Notably, the possible effectors of c-di-GMP were not clear in the strain Sphingomonas sp. WG although its effectors of the other kinds of polysaccharides were investigated before. Therefore, the signal transduction pathway of c-di-GMP will be investigated in next experiment.

Both EPSs and motility are significant contributing factors in biofilm formation. Sphingan-dependent biofilm formation of Sphingomonas sp. strain S2M10 was observed in the presence of specific carbon sources (Czieborowski et al., 2020). Biofilm formation is a stepwise process. For the early stages of biofilm formation, cells transited from motile to a non-motile state for a reversible surface attachment. Then it might form multicellular aggregates based on the swarming motility and the production of EPS such as sphingans to establish the stable association with the substratum. Swarming motility did affect not only the formation but also the architecture of the biofilm. For example, for $P$. aeruginosa, hyper warming motility led to a flat and uniform biofilm, whereas lower swarming motility resulted in microcolonies (Wang et al., 2014). Therefore, WelA might influence the formation of biofilm of Sphingomonas sp. WG due to the lost swarming motility and lower EPS production.

\section{CONCLUSION}

In conclusion, it was obvious that WelA contributes to WL gum biosynthesis and motility of Sphingomonas sp. WG through the branched CckA-ChpT-CtrA pathway and c-di-GMP signaling pathway. A model that features the possible role of WelA in Sphingomonas sp. WG was shown in Figure 6. Therefore, the functions of genes in the CckA-ChpT-CtrA pathway and c-diGMP signaling pathway will be identified in our lab, which will provide more information on the regulation of WL gum biosynthesis and be beneficial for the construction of the WL gum highly producing strains by metabolic engineering.

\section{DATA AVAILABILITY STATEMENT}

The datasets presented in this study can be found in online repositories. The names of the repository/repositories and accession number(s) can be found in the article/ Supplementary Material.

\section{AUTHOR CONTRIBUTIONS}

HL and MC: writing — original draft, writing — review and editing, conceptualization, data curation, and formal analysis. ZZ and BL: methodology and data curation. JL and HX: writing-review and editing, investigation, and data curation. SJ and ZG: investigation and data curation. JW and HZ: conceptualization, supervision, and project administration. All authors contributed to the article and approved the submitted version. 


\section{FUNDING}

This work was financially supported by the National Natural Science Foundation of China (Grant Nos. 31800075 and U1805234), 863 Program (Grant No. 2015AA020925), Program for Innovative Research Team in Science and Technology in Fujian Province University, 100 Talents Program of Fujian Province, Natural Science Foundation of Fujian Province of China, Fundamental Research Funds for the Central Universities and the Development Fund of State Key Laboratory of Heavy

\section{REFERENCES}

Aldridge, P., and Hughes, K. T. (2002). Regulation of flagellar assembly. Curr. Opin. Microbiol. 5, 160-165. doi: 10.1016/S1369-5274(02)00302-8

Alexa, A., Rahnenführer, J., and Lengauer, T. (2006). Improved scoring of functional groups from gene expression data by decorrelating GO graph structure. Bioinformatics 22, 1600-1607. doi: 10.1093/bioinformatics/btl140

Altschul, S. F., Gish, W., Miller, W., Myers, E. W., and Lipman, D. J. (1990). Basic local alignment search tool. J. Mol. Biol. 215, 403-410. doi: 10.1016/S00222836(05)80360-2

Anders, S., and Huber, W. (2010). Differential expression analysis for sequence count data. Genome Biol. 11:R106. doi: 10.1186/gb-2010-11-10-r106

Boyd, C. D., and O’Toole, G. A. (2012). Second messenger regulation of biofilm formation: breakthroughs in understanding c-di-GMP effector systems. Annu. Rev. Cell Dev. Biol. 28, 439-462. doi: 10.1146/annurev-cellbio-101011- 155705

Brilli, M., Fondi, M., Fani, R., Mengoni, A., Ferri, L., Bazzicalupo, M., et al. (2010). The diversity and evolution of cell cycle regulation in alpha-proteobacteria: a comparative genomic analysis. BMC Syst. Biol. 4:52. doi: 10.1186/1752-05 09-4-52

Bu, D., Luo, H., Huo, P., Wang, Z., Zhang, S., He, Z., et al. (2021). KOBAS-i: intelligent prioritization and exploratory visualization of biological functions for gene enrichment analysis. Nucleic Acids Res. 49, W317-W325. doi: 10.1093/ nar/gkab447

Christensen, S., and Serbus, L. R. (2015). Comparative analysis of Wolbachia genomes reveals streamlining and divergence of minimalist two-component systems. G3 5, 983-996. doi: 10.1534/g3.115.017137

Coleman, R. J., Patel, Y. N., and Harding, N. E. (2008). Identification and organization of genes for diutan polysaccharide synthesis from Sphingomonas sp. ATCC 53159. J. Ind. Microbiol. Biotechnol. 35, 263-274. doi: 10.1007/ s10295-008-0303-3

Czieborowski, M., Hubenthal, A., Poehlein, A., Vogt, I., and Philipp, B. (2020). Genetic and physiological analysis of biofilm formation on different plastic surfaces by Sphingomonas sp. strain S2M10 reveals an essential function of sphingan biosynthesis. Microbiology 166, 918-935. doi: 10.1099/mic.0.000961

Dardis, C., Quelas, J. I., Mengucci, F., Althabegoiti, M. J., Lodeiro, A. R., Mongiardini, E. J., et al. (2021). Dual control of flagellar synthesis and exopolysaccharide production by FlbD-FliX class II regulatory proteins in Bradyrhizobium diazoefficiens. J. Bacteriol. 203, e00403-e00420. doi: 10.1128/ JB.00403-20

Fialho, A. M., Moreira, L. M., Granja, A. T., Popescu, A. O., Hoffmann, K., and SaCorreia, I. (2008). Occurrence, production, and applications of gellan: current state and perspectives. Appl. Microbiol. Biotechnol. 79, 889-900. doi: 10.1007/ s00253-008-1496-0

Floyd, K. A., Lee, C. K., Xian, W., Nametalla, M., Valentine, A., Crair, B., et al. (2020). c-di-GMP modulates type IV MSHA pilus retraction and surface attachment in Vibrio cholerae. Nat. Commun. 11:1549. doi: 10.1038/s41467020-15331-8

Francez-Charlot, A., Kaczmarczyk, A., and Vorholt, J. A. (2015). The branched CcsA/CckA-ChpT-CtrA phosphorelay of Sphingomonas melonis controls motility and biofilm formation. Mol. Microbiol. 97, 47-63. doi: 10.1111/mmi. 13011

Freitas, F., Alves, V. D., and Reis, M. A. (2011). Advances in bacterial exopolysaccharides: from production to biotechnological applications. Trends Biotechnol. 29, 388-398. doi: 10.1016/j.tibtech.2011.03.008
Oil Processing (Grant No. 20CX02202A), Engineering Research Center of Industrial Biocatalysis, Fujian Province Universities (Grant No. ERCIB2020-01).

\section{SUPPLEMENTARY MATERIAL}

The Supplementary Material for this article can be found online at: https://www.frontiersin.org/articles/10.3389/fmicb. 2022.792315/full\#supplementary-material

Geourjon, C., and Deleage, G. (1995). SOPMA: significant improvements in protein secondary structure prediction by consensus prediction from multiple alignments. Comput. Appl. Biosci. 11, 681-684. doi: 10.1093/bioinformatics/11. 6.681

Harding, N. E., Patel, Y. N., and Coleman, R. J. (2004). Organization of genes required for gellan polysaccharide biosynthesis in Sphingomonas elodea ATCC 31461. J. Ind. Microbiol. Biotechnol. 31, 70-82. doi: 10.1007/s10295-0040118-9

Johnson, D. R., Coronado, E., Moreno-Forero, S. K., Heipieper, H. J., and van der Meer, J. R. (2011). Transcriptome and membrane fatty acid analyses reveal different strategies for responding to permeating and non-permeating solutes in the bacterium Sphingomonas wittichii. BMC Microbiol. 11:250. doi: 10.1186/ 1471-2180-11-250

Jung, Y. C., Lee, M. A., Kim, H. S., and Lee, K. H. (2021). Role of DegQ in differential stability of flagellin subunits in Vibrio vulnificus. Npj Biofilms Microbiomes 7:32. doi: 10.1038/s41522-021-00206-7

Koppenhofer, S., and Lang, A. S. (2020). Interactions among Redox Regulators and the CtrA Phosphorelay in Dinoroseobacter shibae and Rhodobacter capsulatus. Microorganisms 8:562. doi: 10.3390/microorganisms8040562

Kovach, M. E., Elzer, P. H., Hill, D. S., Robertson, G. T., Farris, M. A., Roop, R. M. II, et al. (1995). Four new derivatives of the broad-host-range cloning vector pBBR1MCS, carrying different antibiotic-resistance cassettes. Gene 166, 175-176. doi: 10.1016/0378-1119(95)00584-1

Kumar, A. S., Mody, K., and Jha, B. (2007). Bacterial exopolysaccharidesa perception. J. Basic Microbiol. 47, 103-117. doi: 10.1002/jobm.20061 0203

Kumar, S., Stecher, G., and Tamura, K. (2016). MEGA7: molecular evolutionary genetics analysis version 7.0 for bigger datasets. Mol. Biol. Evol. 33, 1870-1874. doi: $10.1093 / \mathrm{molbev} / \mathrm{msw} 054$

Langmead, B., and Salzberg, S. L. (2012). Fast gapped-read alignment with Bowtie 2. Nat. Methods 9, 357-359. doi: 10.1038/nmeth.1923

Larkin, M. A., Blackshields, G., Brown, N. P., Chenna, R., McGettigan, P. A., McWilliam, H., et al. (2007). Clustal W and Clustal X version 2.0. Bioinformatics 23, 2947-2948. doi: 10.1093/bioinformatics/btm404

Laub, M. T., McAdams, H. H., Feldblyum, T., Fraser, C. M., and Shapiro, L. (2000). Global analysis of the genetic network controlling a bacterial cell cycle. Science 290, 2144-2148. doi: 10.1126/science.290.5499.2144

Lee, S. Y., Ahn, J. Y., Kim, M., Sekhon, S. S., Cho, S. J., Kim, Y. C., et al. (2017). Phenotypic and proteomic analysis of positively regulated gellan biosynthesis pathway in Sphingomonas elodea. Anim. Cells Syst. 21, 115-123. doi: 10.1080/ 19768354.2017.1290678

Lee, Y. L., Xu, X. R., Wallenstein, S., and Chen, J. (2009). Gene expression profiles of the one-carbon metabolism pathway. J. Genet. Genomics 36, 277-282. doi: 10.1016/S1673-8527(08)60115-0

Lelchat, F., Cozien, J., Le Costaouec, T., Brandilly, C., Schmitt, S., Baudoux, A. C., et al. (2015). Exopolysaccharide biosynthesis and biodegradation by a marine hydrothermal Alteromonas sp. strain. Appl. Microbiol. Biotechnol. 99, 2637-2647. doi: 10.1007/s00253-014-6075-y

Letunic, I., Doerks, T., and Bork, P. (2015). SMART: recent updates, new developments and status in 2015. Nucleic Acids Res. 43, D257-D260. doi: 10. 1093/nar/gku949

Li, H., Jiao, X., Sun, Y., Sun, S., Feng, Z., Zhou, W., et al. (2016b). The preparation and characterization of a novel sphingan WL from marine Sphingomonas sp. WG. Sci. Rep. 6:37899. doi: 10.1038/srep37899 
Li, H., Feng, Z. M., Sun, Y. J., Zhou, W. L., Jiao, X., and Zhu, H. (2016a). Draft Genome sequence of Sphingomonas sp. WG, a Welan gum-producing strain. Genome Announc. 4:e1709-15. doi: 10.1128/genomeA.01709-15

Li, H., Li, J., Jiao, X., Li, K. H., Sun, Y. J., Zhou, W. L., et al. (2019). Characterization of the biosynthetic pathway of nucleotide sugar precursor UDP-glucose during sphingan WL gum production in Sphingomonas sp. WG. J. Biotechnol. 302, 1-9. doi: 10.1016/j.jbiotec.2019.06.005

Li, H., Li, J., Zhou, W., Jiao, X., Sun, Y., Shen, Y., et al. (2018). An efficient production of a novel carbohydrate polymer Sphingan WL. J. Chem. Technol. Biotechnol. 93, 3472-3482. doi: 10.1002/jctb.5705

Li, H., Li, K., Guo, Z., Xue, H., Li, J., Ji, S., et al. (2021). The Function of beta-1,4Glucuronosyltransferase WelK in the Sphingan WL Gum Biosynthesis Process in Marine Sphingomonas sp. WG. Mar. Biotechnol. 23, 39-50. doi: 10.1007/ s10126-020-09998-9

Lyon, P., Strippoli, V., Fang, B., and Cimmino, L. (2020). B vitamins and onecarbon metabolism: implications in human health and disease. Nutrients 12:2867. doi: 10.3390/nu12092867

Maruyama, Y., Kobayashi, M., Murata, K., and Hashimoto, W. (2015). Formation of a single polar flagellum by two distinct flagellar gene sets in Sphingomonas sp. strain A1. Microbiology 161, 1552-1560. doi: 10.1099/mic.0.000119

Mattos-Graner, R. O., and Duncan, M. J. (2017). Two-component signal transduction systems in oral bacteria. J. Oral Microbiol. 9:1400858. doi: 10.1080/ 20002297.2017.1400858

Minamino, T., Kinoshita, M., Morimoto, Y. V., and Namba, K. (2021). The FlgN chaperone activates the $\mathrm{Na}(+)$-driven engine of the Salmonella flagellar protein export apparatus. Commun. Biol. 4:335. doi: 10.1038/s42003-021-01865-0

Minnich, S. A., Ohta, N., Taylor, N., and Newton, A. (1988). Role of the 25-, 27-, and 29-kilodalton flagellins in Caulobacter crescentus cell motility: method for construction of deletion and Tn5 insertion mutants by gene replacement. J. Bacteriol. 170, 3953-3960. doi: 10.1128/jb.170.9.3953-3960.1988

Newell, P. D., Yoshioka, S., Hvorecny, K. L., Monds, R. D., and O’Toole, G. A. (2011). Systematic analysis of diguanylate cyclases that promote biofilm formation by Pseudomonas fluorescens Pf0-1. J. Bacteriol. 193, 4685-4698. doi: 10.1128/JB.05483-11

Overhage, J., Lewenza, S., Marr, A. K., and Hancock, R. E. (2007). Identification of genes involved in swarming motility using a Pseudomonas aeruginosa PAO1 mini-Tn5-lux mutant library. J. Bacteriol. 189, 2164-2169. doi: 10.1128/jb. 01623-06

Pelicic, V., Reyrat, J. M., and Gicquel, B. (1996). Generation of unmarked directed mutations in mycobacteria, using sucrose counter-selectable suicide vectors. Mol. Microbiol. 20, 919-925. doi: 10.1111/j.1365-2958.1996.tb02533.x

Pini, F., De Nisco, N. J., Ferri, L., Penterman, J., Fioravanti, A., Brilli, M., et al. (2015). Cell cycle control by the master regulator CtrA in Sinorhizobium meliloti. PLoS Genet. 11:e1005232. doi: 10.1371/journal.pgen.1005232

Rivera-Osorio, A., Osorio, A., Poggio, S., Dreyfus, G., and Camarena, L. (2018). Architecture of divergent flagellar promoters controlled by CtrA in Rhodobacter sphaeroides. BMC Microbiol. 18:129. doi: 10.1186/s12866-018-1264-y

Sa-Correia, I., Fialho, A. M., Videira, P., Moreira, L. M., Marques, A. R., and Albano, H. (2002). Gellan gum biosynthesis in Sphingomonas paucimobilis ATCC 31461: genes, enzymes and exopolysaccharide production engineering. J. Ind. Microbiol. Biotechnol. 29, 170-176. doi: 10.1038/sj.jim.7000266

Sánchez-Romero, M. A., and Casadesús, J. (2020). The bacterial epigenome. Nat. Rev. Microbiol. 18, 7-20. doi: 10.1038/s41579-019-0286-2

Schmid, J., Ruhmann, B., Sieber, V., Romero-Jimenez, L., Sanjuan, J., and PerezMendoza, D. (2018). Screening of c-di-GMP-regulated exopolysaccharides in host interacting bacteria. Methods Mol. Biol. 1734, 263-275. doi: 10.1007/9781-4939-7604-1_21

Schmid, J., Sieber, V., and Rehm, B. (2015). Bacterial exopolysaccharides: biosynthesis pathways and engineering strategies. Front. Microbiol. 6:496. doi: 10.3389/fmicb.2015.00496

Schmid, J., Sperl, N., and Sieber, V. (2014). A comparison of genes involved in sphingan biosynthesis brought up to date. Appl. Microbiol. Biotechnol. 98, 7719-7733. doi: 10.1007/s00253-014-5940-z

Skerker, J. M., Prasol, M. S., Perchuk, B. S., Biondi, E. G., and Laub, M. T. (2005). Two-component signal transduction pathways regulating growth and cell cycle progression in a bacterium: a system-level analysis. PLoS Biol. 3:e334. doi: 10.1371/journal.pbio.0030334
Smith, T. G., and Hoover, T. R. (2009). Deciphering bacterial flagellar gene regulatory networks in the genomic era. Adv. Appl. Mircobiol. 67, 257-295. doi: 10.1016/S0065-2164(08)01008-3

Spangler, C., Bohm, A., Jenal, U., Seifert, R., and Kaever, V. (2010). A liquid chromatography-coupled tandem mass spectrometry method for quantitation of cyclic di-guanosine monophosphate. J. Microbiol. Methods 81, 226-231. doi: 10.1016/j.mimet.2010.03.020

Thorne, L., Mikolajczak, M. J., Armentrout, R. W., and Pollock, T. J. (2000). Increasing the yield and viscosity of exopolysaccharides secreted by Sphingomonas by augmentation of chromosomal genes with multiple copies of cloned biosynthetic genes. J. Ind. Microbiol. Biotechnol. 25, 49-57. doi: 10.1038/ sj.jim.7000019

Tsang, J., and Hoover, T. R. (2014). Themes and variations: regulation of RpoN-dependent flagellar genes across diverse bacterial species. Scientifica 2014:681754. doi: 10.1155/2014/681754

Vega-Baray, B., Domenzain, C., Rivera, A., Alfaro-López, R., Gómez-César, E., Poggio, S., et al. (2015). The flagellar set Fla2 in Rhodobacter sphaeroides is controlled by the CckA pathway and is repressed by organic acids and the expression of Fla1. J. Bacteriol. 197, 833-847. doi: 10.1128/jb.02429-14

Wang, S. W., Yu, S., Zhang, Z. Y., Wei, Q., Yan, L., Ai, G. M., et al. (2014). Coordination of swarming motility, biosurfactant synthesis, and biofilm matrix exopolysaccharide production in Pseudomonas aeruginosa. Appl. Environ. Microb. 80, 6724-6732. doi: 10.1128/Aem.01237-14

Wilkins, M. R., Gasteiger, E., Bairoch, A., Sanchez, J. C., Williams, K. L., Appel, R. D., et al. (1999). Protein identification and analysis tools in the ExPASy server. Methods Mol. Biol. 112, 531-552. doi: 10.1385/1-59259-584-7:531

Willett, J. W., Herrou, J., Briegel, A., Rotskoff, G., and Crosson, S. (2015). Structural asymmetry in a conserved signaling system that regulates division, replication, and virulence of an intracellular pathogen. Proc. Natl. Acad. Sci. U. S. A. 112, E3709-E3718. doi: 10.1073/pnas.1503118112

Willis, L. M., and Whitfield, C. (2013). Structure, biosynthesis, and function of bacterial capsular polysaccharides synthesized by $\mathrm{ABC}$ transporterdependent pathways. Carbohyd. Res. 378, 35-44. doi: 10.1016/j.carres.2013. 05.007

Xu, X. P., Nie, Z. M., Zheng, Z. Y., Zhu, L., Zhang, H. T., and Zhan, X. B. (2017). Different nitrogen sources change the transcriptome of welan gum-producing strain Sphingomonas sp ATCC 31555. Arch. Microbiol. 199, 1055-1064. doi: 10.1007/s00203-017-1372-3

Yamazaki, M., Thorne, L., Mikolajczak, M., Armentrout, R. W., and Pollock, T. J. (1996). Linkage of genes essential for synthesis of a polysaccharide capsule in Sphingomonas strain S88. J. Bacteriol. 178, 2676-2687. doi: 10.1128/jb.178.9. 2676-2687.1996

Zhou, B., Schrader, J. M., Kalogeraki, V. S., Abeliuk, E., Dinh, C. B., Pham, J. Q., et al. (2015). The global regulatory architecture of transcription during the Caulobacter cell cycle. PLoS Genet. 11:e1004831. doi: 10.1371/journal.pgen. 1004831

Zhou, W. L., Jiao, X., Sun, Y. J., Li, H., and Zhu, H. (2017). Determination of extracellular polysaccharides produced by marine derived Sphingomonas sp. WG. Chin. J. Mar. Drugs 36, 27-34.

Conflict of Interest: The authors declare that the research was conducted in the absence of any commercial or financial relationships that could be construed as a potential conflict of interest.

Publisher's Note: All claims expressed in this article are solely those of the authors and do not necessarily represent those of their affiliated organizations, or those of the publisher, the editors and the reviewers. Any product that may be evaluated in this article, or claim that may be made by its manufacturer, is not guaranteed or endorsed by the publisher.

Copyright (C) $2022 \mathrm{Li}$, Chen, Zhang, Li, Liu, Xue, Ji, Guo, Wang and Zhu. This is an open-access article distributed under the terms of the Creative Commons Attribution License (CC BY). The use, distribution or reproduction in other forums is permitted, provided the original author(s) and the copyright owner(s) are credited and that the original publication in this journal is cited, in accordance with accepted academic practice. No use, distribution or reproduction is permitted which does not comply with these terms. 